\title{
Uma Abordagem SDN para Priorização de Tráfego em Ambientes Hospitalares Inteligentes
}

\author{
Lucas B. Oliveira ${ }^{1}$, Robertson Lima ${ }^{1}$, Danilo F. S. Santos ${ }^{3}$, \\ Paulo E. S. Barbosa ${ }^{2}$, Fernando Matos ${ }^{1}$ \\ ${ }^{1}$ Centro de Informática - Universidade Federal da Paraíba \\ João Pessoa, PB - Brasil \\ ${ }^{2}$ Núcleo de Tecnologias Estratégicas em Saúde - NUTES \\ Universidade Estadual da Paraíba (UEPB) - Campina Grande, PB - Brasil \\ ${ }^{3}$ Departamento de Engenharia Elétrica \\ Universidade Federal de Campina Grande (UFCG) - Campina Grande, PB - Brasil \\ lucas.barbosa@ppgi.ci.ufpb.br, robertsonlima91@gmail.com, \\ danilo.santos@dee.ufcg.edu.br, paulo.barbosa@nutes.uepb.edu.br, \\ fernando@ci.ufpb.br
}

\begin{abstract}
Nowadays, several critical, non-critical medical devices and health IT systems are being developed for sharing medical data. Such devices can also share the same network and be interconected to systems to perform monitoring and analysis of clinical data. Due to this centralization and sharing of network resources, priority traffic of critical medical devices can be harmed. Therefore, this paper proposes an SDN-based solution for the management of a hospital network through the prioritization of critical device traffic. This solution aims to meet the quality parameters required by each service in smart hospital environments.
\end{abstract}

Resumo. Recentemente, uma grande quantidade de dispositivos médicos conectados e sistemas de TI destinados à saúde estão sendo desenvolvidos $e$ integrados para o compartilhamento de dados. Tais dispositivos e sistemas compartilham a mesma rede, podendo ser acoplados a sistemas de monitoramento e análise de dados clínicos. Em decorrência deste compartilhamento, tráfegos prioritários de dispositivos médicos podem ser prejudicados por congestionamentos de rede. Nesse cenário, este artigo propõe uma solução baseada em SDN (Software Defined Networks) para o gerenciamento de uma rede hospitalar através da priorização de tráfego para dispositivos médicos prioritários. Esta solução busca a observância dos parâmetros de qualidade exigidos por cada serviço em um ambiente de hospital inteligente.

\section{Introdução}

Ambientes hospitalares inteligentes caracterizam-se pela presença concomitante de diversos dispositivos médicos e não médicos que podem interagir entre si e que muitas vezes compartilham a mesma rede. Apesar de ser uma solução que reduz custos, este compartilhamento pode ocasionar disputa por recursos de rede. Outra característica atual de tais ambientes hospitalares é a utilização crescente de plataformas integradas que facilitam o monitoramento e comunicação de dispositivos médicos. Uma destas plataformas que está em desenvolvimento e é amplamente disseminada é o Open-source Integrated Clinical 
Environment ${ }^{1}$ (OpenICE), o qual é a implementação de um Ambiente Clínico Integrado (Integrated Clinical Environment - ICE) como descrito na norma [ASTM F2761 2009]. O OpenICE é visto como uma plataforma de sistema distribuído que monitora diversos dispositivos médicos através de uma rede local, na qual reside uma central de monitoramento que realiza a concentração e análise dos dados destes dispositivos [Almadani et al. 2016].

Contudo, devido a centralização de dados para o monitoramento, e do compartilhamento da rede por outros equipamentos além dos dispositivos médicos, podem ocorrer problemas de disponibilização de recursos e priorização de tráfego em cenários hospitalares, onde a geração e o compartilhamento de dados pode atingir grandes volumes [Arney et al. 2012]. Neste cenário o tráfego com requisitos rígidos, tais como os gerados por dispositivos médicos críticos em situações de alarmes, pode não atingir os parâmetros de qualidade de serviço exigidos em tais situações. Dependendo do contexto ao qual o dispositivo está atuando, isto pode gerar um atraso crucial no atendimento ao paciente.

Devido a isto, diversas tecnologias acopladas à tais sistemas de gerenciamento de dispositivos médicos estão emergindo com intuito de proporcionar uma melhor qualidade de serviço, necessária para o pleno uso desses equipamentos no contexto da saúde [Gomez-Sacristan et al. 2015]. Software Defined Networking (SDN) é uma abordagem promissora que já vêm sendo utilizada para facilitar e automatizar o gerenciamento de redes de computadores e que pode ser aplicada em ambientes hospitalares. Em SDN, há a separação do plano de dados (realizado por equipamentos, tais como switches e roteadores) do plano de controle (realizado por um software controlador). O controlador é a entidade que possui a visão completa da rede e é responsável por tomar as decisões de encaminhamento dos pacotes. Desta forma, o gerenciamento da rede é realizado todo por software, o que facilita a configuração e a aplicação de múltiplas políticas de gerenciamento com o intuito de lidar com diversos cenários de comportamento da rede [Hu 2015].

Portanto, este artigo tem por objetivo propor uma solução baseada em SDN para o gerenciamento de redes hospitalares. Esta solução visa priorizar o tráfego de equipamentos que demandem qualidade de serviço em detrimento daqueles tolerantes a atraso. Para a validação da solução foram realizados testes com tráfego gerado tanto por equipamentos reais quanto simulados de modo a caracterizar o problema. O restante deste artigo está estruturado como descrito a seguir: A Seção 2 discute os trabalhos relacionados. A Seção 3 descreve a solução proposta juntamente com a metodologia utilizada para a validação e os resultados de testes preliminares. Finalmente, a Seção 4 conclui o artigo.

\section{Trabalhos Relacionados}

Muitos são os trabalhos que tangenciam este tema com o intuito de avançar na modernização de centros hospitalares, como no trabalho de [Arney et al. 2012], onde é relatado a complexidade dentro de salas de operações partindo de um cenário simples para um mais complexo. No primeiro a taxa de dados que trafega entre os dispositivos médicos é baixa e de fácil administração. Entretanto, ao considerar cenários mais complexos onde estão inseridos dispositivos médicos capazes de realizar a exportação e importação de imagens no formato DICOM (do inglês, Digital Imaging and Communications in Medicine) percebe-se o grande impacto que é gerado no desempenho da rede, como nos experimentos realizados, os quais consideram redes que disponham de uma largura de banda de $100 \mathrm{Mbps}, 500 \mathrm{Mbps}$ e $1 \mathrm{Gbps}$. Nestes experimentos constatou-se a degradação parcial ou completa da rede ao considerar a implantação de mais de duas salas de opera-

\footnotetext{
${ }^{1}$ https://www.openice.info/
} 
ções avançadas, nas quais além de admitirem dispositivos médicos também possuíam uma workstation DICOM para a transferência de imagens, podendo assim gerar uma alta taxa de dados na rede, impossibilitando o tráfico originado nos dispositivos médicos críticos.

Segundo o trabalho de [Pahontu et al. 2015] é demonstrado a relevância do compartilhamento de dados de dispositivos médicos com sistemas integrados de saúde, para isso é considerado uma arquitetura baseada na implementação de um gateway responsável pela conexão entre esses dois componentes. Neste ainda é visto a implantação de uma sala de operações, a qual faz uso de serviços de imagens no formato DICOM para o compartilhamento dos exames médicos. Em termos gerais, este trabalho trata da estruturação dos componentes do gateway, juntamente com outros serviços que possibilitem a integração de sistemas de TI com os dispositivos médicos, e a interoperabilidade destes existente dentro de uma rede local em uma sala de operações clínicas.

Por fim, assim como neste trabalho, requisitos referente a qualidade de serviço (QoS) prestados aos componentes médicos são discutidos nos trabalhos de [Hu 2015] e [Gomez-Sacristan et al. 2015]. No primeiro é realizado uma proposta de arquitetura intitulada de Software Defined Healthcare Network com o intuito de projetar um controlador centralizado para gerenciamento de dispositivos físicos e o fornecimento de uma interface para coleta, transmissão e processamento de dados. Já no segundo, é realizado um estudo com o intuito de estipular o consumo da banda por parte dos serviços existentes em um hospital inteligente. A partir do qual, constatou-se uma possível categorização dos próprios serviços previsto, em relação a taxa de transferência dos dados.

\section{Solução Proposta}

Como descrito anteriormente, a solução proposta objetiva melhorar o gerenciamento de redes hospitalares ao dar suporte à priorização de tráfego de dispositivos médicos através de SDN. Considerando, pois, ambientes hospitalares inteligentes, os quais são compostos por diversas salas de operação avançadas que contêm vários dispositivos médicos críticos, pessoais e operacionais. O tráfego gerado por estes dispositivos é enviado para a central de monitoramento, onde é hospedado aplicativos que realizam a análise dos dados, e gerenciam interfaces externas para recursos hospitalares, como sistemas de prontuário médico eletrônico[Arney et al. 2017]. A Figura 1 apresenta a arquitetura da solução proposta integrada a um cenário hospitalar inteligente, o qual possui os seguintes componentes:

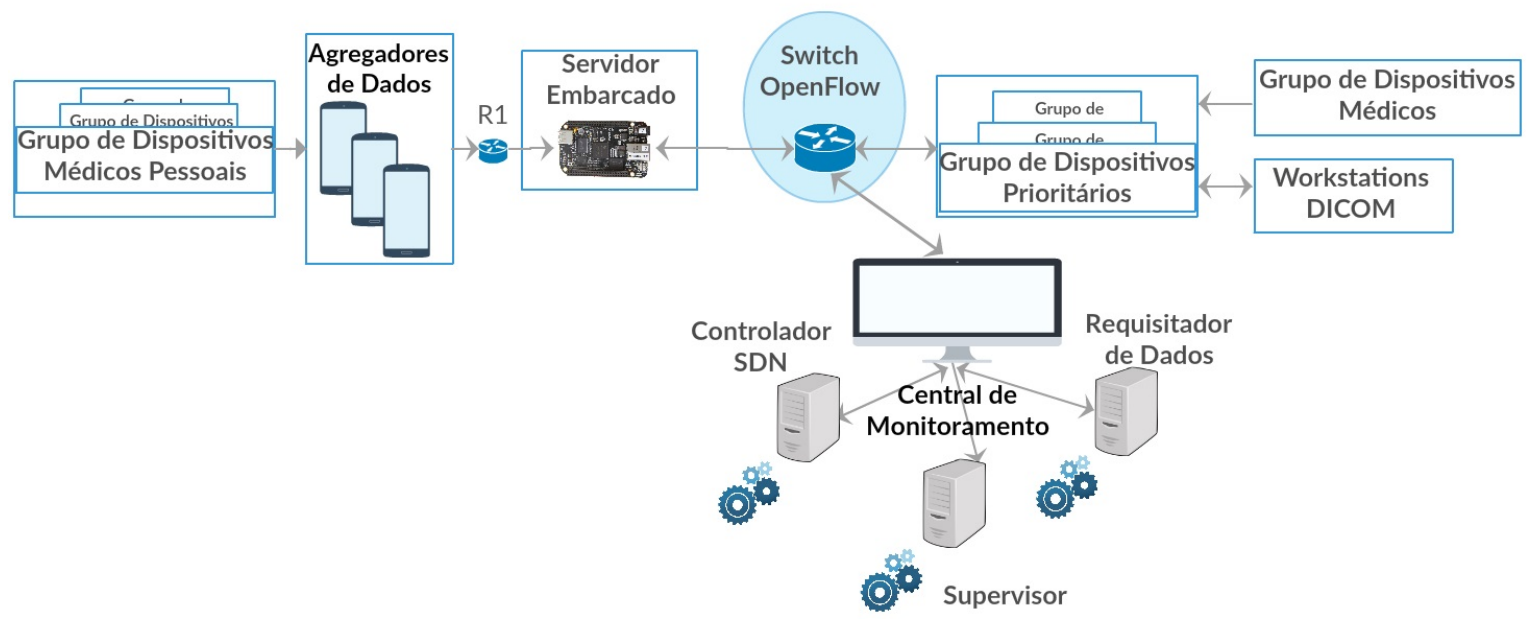

Figura 1. Arquitetura da Implantação da Rede de Dispositivos Médicos com SDN 
- Grupo de Dispositivos Prioritários - são todos os itens classificados como dispositivos médicos críticos. Podem ser utilizados por um grupo de pacientes, ou um único paciente que pode utilizar diversos dispositivos médicos conectados simultaneamente. Este grupo é formado por dois sub-grupos: (i) dispositivos médicos críticos, que capturam os dados vitais de pacientes de forma contínua ou periódica; (ii) equipamentos específicos de imagens médicas, os quais são utilizados para gerar e compartilhar imagens clínicas de alta resolução.

- Grupo de Dispositivos Médicos Pessoais - Este representa diversos dispositivos não-críticos que podem ser integrados com tecnologia sem-fio como Bluetooth ou Wifi, e seguem padrões de interoperabilidade para o monitoramento da saúde pessoal, como por exemplo, o protocolo IEEE 11073. Estes dispositivos estão presentes em sistemas HealthCare, os quais realizam o compartilhamento de dados dos dispositivos médicos pessoais com serviços e sistemas de TI de saúde, através de um Hub que os conecta à rede hospitalar ou a um ambiente clínico integrado.

- Agregador de Dados - O agregador é uma aplicativo residente em dispositivos móveis utilizado para estabelecer a comunicação e coletar os dados gerados pelos diversos dispositivos médicos pessoais. Tal comunicação entre os dispositivos de saúde e o agregador pode ser das mais variadas, entretanto umas das tecnologias mais utilizadas é a comunicação Bluetooth em consequência do mesmo estar presente nos mais modernos dispositivos médicos pessoais.

- Servidor Embarcado - O servidor realiza a centralização dos dados coletados dos diversos agregadores de dados. A comunicação entre o servidor e a aplicação do dispositivo móvel é realizado através de uma API REST. Em outros cenários, o servidor e o agregador podem ser executados em um mesmo equipamento. $\mathrm{O}$ intuito de realizar a concentração dos dados no servidor faz-se necessário para controlar a inserção desses dados de menor prioridade na rede. Com isto, os dados armazenados no servidor poderão ser posteriormente requisitado pela Central de Monitoramento em um momento oportuno. Este componente foi implementado em uma plataforma embarcada BeagleBone Black RevC, com um sistema operacional embarcado Linux, executando um servidor web apto a receber requisições REST para realizar a manipulação e consultas em um banco de dados SQLite 3.0.

- Switches OpenFlow - Equipamento utilizado para realizar o repasse e controle dos pacotes emitidos na rede. Tal controle é realizado através da manipulação da tabela de fluxo, de acordo com as políticas definidas pelo controlador, conforme o perfil do tráfego na rede. Durante os experimentos de validação será utilizado o roteador Tplink, modelo TL-WR1043ND, versão 3.

- Central de Monitoramento - É um dos principais componentes da solução e é composto por três módulos: (i) controlador SDN, que é o responsável por avaliar o perfil do tráfego da rede e instalar os fluxos no switch; (ii) requisitador de dados, que tem por objetivo solicitar ao servidor embarcado os dados dos dispositivos médicos pessoais, coletados e enviados anteriormente através dos agregadores de dados; (iii) supervisor, onde residirão os aplicativos de análise, exibição e exportação de todos os dados dos dispositivos médicos emitidos através da rede.

\subsection{Funcionamento}

O funcionamento da solução proposta pode ser descrito como se segue. Em um cenário de atenção, os dispositivos prioritários irão enviar dados vitais dos pacientes bem como as imagens de alta resolução diretamente para a central de monitoramento. Durante o envio desses dados, a central de monitoramento analisará se alarmes estão sendo emitidos por 
esses dispositivos médicos prioritários, e se o atraso de recepção desses dados está dentro dos parâmetros de QoS definidos para o sistema [Arney et al. 2012]. Caso um alarme seja identificado ou os parâmetros de QoS estejam prejudicados, a central implanta regras de SDN que gerenciarão o tráfego de dados dos Switchs na rede. Essas regras irão priorizar o tráfego desses dispositivos em contra-ponto aos dispositivos médicos pessoais ou outros sistemas da rede. Esta execução de regras é de responsabilidade do módulo Controlador SDN que reside na Central de Monitoramento. Por fim, de modo a não bloquear o total funcionamento dos outros dispositivos médicos, a central de monitoramento pode realizar a requisição dos dados sob-demanda ao servidor embarcado, de modo que o mesmo funcione como relay de dados na rede. Deste modo, a central pode controlar o fluxo de recepção dos dados destes dispositivos médicos pessoais, sem que os mesmos impactem o fluxo de dados prioritários durante situações de atenção.

\subsection{Metodologia de validação}

A abordagem para validação do experimento será realizada em cima do cenário anterior, a partir da implantação de um ambiente com um switch OpenFlow com a capacidade de realizar taxas de transferência de até 1 Gbps. Desta maneira, será realizado de forma gradativa a inserção de salas de operações avançadas para demonstrar o impacto do tráfego gerado na rede. Serão realizados testes com e sem a solução SDN proposta de forma a validar e avaliar sua eficácia. Nestes testes serão utilizados como valores base parâmetros já consolidados em outros experimentos, como por exemplo, o tempo máximo para a transferência de dados provindos de dispositivos médicos críticos e da comunicação referente à transferência de imagem no formato DICOM, os quais foram definidos como 1 segundo e 20 segundos respectivamente, como apresentado em [Arney et al. 2012].

\subsection{Avaliação do Problema}

Foram realizados testes preliminares para verificar o tráfego gerado por dispositivos médicos críticos na rede. Nestes testes foram utilizados simuladores de quatro dispositivos presentes no OpenICE, os quais foram: (i) monitor multiparamétrico, (ii) oxímetro, (iii) eletrocardiograma e (iv) capnômetro. Como pode ser visto na Figura 2(a), os dispositivos produziram uma taxa média de aproximadamente $331 \mathrm{Kbps}$ durante 30 segundos de funcionamento, alcançando picos de até $1,768 \mathrm{Mbps}$. Outro teste foi realizado para verificar o tráfego gerado por requisições ao banco de dados presente no servidor embarcado (Figura 2(b)). Foram disparadas cinco requisições pela central de monitoramento ao banco de dados, gerando uma média de $156 \mathrm{Kbps}$, alcançando picos de $369 \mathrm{Kbps}$. A partir disto, observa-se que o crescimento no número de dispositivos, bem como a inclusão das workstations DICOM, as quais podem alcançar taxas de transferência de até 800 Mbps [Arney et al. 2012], possivelmente tornará instável e inviabilizará a transferência de dados dos dispositivos médicos críticos.

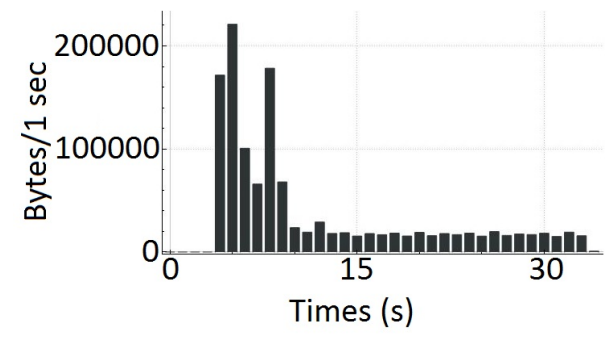

(a)

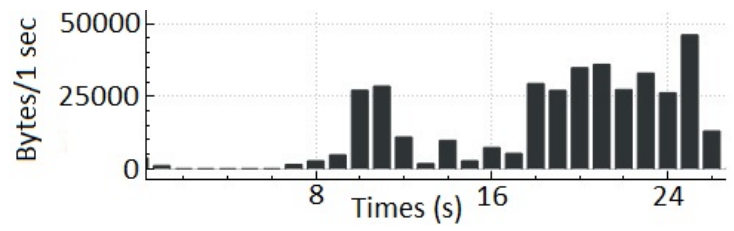

(b)

Figura 2. Resultados dos Experimentos 
Os resultados observados nos testes preliminares atestaram o problema de priorização de rede em um ambiente clínico integrado. Em uma implementação preliminar, a priorização de tráfego de rede para dispositivos em um grupo prioritário mostrou eficácia em diminuir o atraso médio na entrega de dados prioritários, como alarmes.

\section{Considerações Finais}

Este artigo propôs uma solução baseada em SDN para o gerenciamento de um ambiente hospitalar inteligente através da priorização de tráfego de dispositivos médicos críticos. Tal priorização considera os parâmetros definidos por cada serviço para atender à qualidade exigida. Foram realizados testes preliminares para verificar a taxa de envio de quatro dispositivos médicos e de consultas ao banco de dados no servidor embarcado. No futuro, os resultados da avaliação do problema servirão de parâmetros para a configuração dos casos de uso a serem testados para validar e avaliar a solução proposta de modo completo. Tais casos de uso considerarão diversos aspectos relacionados a hospitais inteligentes e salas de operações clínicas avançadas. Por fim, a infra-estrutura desenvolvida nesse projeto está sendo reusada no Projeto OCARIoT (ocariot.com), que usa o potencial da IoT para desenvolver planos de aconselhamento em obesidade para crianças e educadores.

\section{Agradecimentos.}

Essa pesquisa está sendo conduzida com recursos provenientes de apoio da CAPES (Coordenação de Aperfeiçoamento de Pessoal de Nível Superior) e do Projeto OCARIoT, financiado pelo programa da União Européia HORIZON 2020, ID 777082, e pela Rede Nacional de Ensino e Pesquisa (RNP), ID 003008.

\section{Referências}

Almadani, B., Saeed, B., and Alroubaiy, A. (2016). Healthcare systems integration using Real Time Publish Subscribe (RTPS) middleware. Computers and Electrical Engineering, 50:67-78.

Arney, D., Goldman, J. M., Bhargav-Spantzel, A., Basu, A., Taborn, M., Pappas, G., and Robkin, M. (2012). Simulation of medical device network performance and requirements for an integrated clinical environment. Biomedical instrumentation \& technology / Association for the Advancement of Medical Instrumentation, 46(4):308-315.

Arney, D., Plourde, J., and Goldman, J. M. (2017). OpenICE medical device interoperability platform overview and requirement analysis. Biomedizinische Technik, 63(1):39_ 47.

ASTM F2761 (2009). Medical Devices and Medical Systems - Essential safety requirements for equipment comprising the patient-centric integrated clinical environment (ICE) - Part 1: General requirements and conceptual model, ASTM International.

Gomez-Sacristan, A., Rodriguez-Hernandez, M. A., and Sempere, V. (2015). Evaluation of Quality of Service in Smart-Hospital Communications. Journal of Medical Imaging and Health Informatics, 5(8):1864-1869.

Hu, L. (2015). Software Defined Healthcare Networ. IEEE Wireless Communications, (December):67-75.

Pahontu, R., Schneider, G., Bergh, B., and Merzweiler, A. (2015). An IHE based gateway architecture to link healthcare IT with medical devices in the operating room. In 2015 17th International Conference on E-Health Networking, Application and Services, HealthCom 2015, pages 586-589. 